Med Klin Intensivmed Notfmed 2021 · 116:168-170 https://doi.org/10.1007/s00063-021-00785-5 Angenommen: 14. Januar 2021

Online publiziert: 3. Februar 2021

(c) Springer Medizin Verlag GmbH, ein Teil von Springer Nature 2021

\section{Guido Michels ${ }^{1}$ - Uta Ochmann ${ }^{2}$}

${ }^{1}$ Klinik für Akut- und Notfallmedizin, St.-Antonius-Hospital Eschweiler, Akademisches Lehrkrankenhaus der RWTH Aachen, Eschweiler, Deutschland

${ }^{2}$ Institut und Poliklinik für Arbeits-, Sozial- und Umweltmedizin, Stabsstelle Betriebsärztlicher Dienst, Ludwig-Maximilians-Universitätsklinikum, München, Deutschland

\title{
Beschäftigung von schwangeren Mitarbeiterinnen im Gesundheitssystem während der zweiten Welle der COVID-19- Pandemie
}

Die Coronavirus-Disease(COVID)-19Pandemie breitet sich immer noch weiter aus. Nach einer kurzen Stabilisierungsphase im Sommer dieses Jahrs befinden wir uns infektionsepidemiologisch aktuell wieder in einer kritischen Situation bzw. in der sog. 2. Welle. Das Personal im Bereich der Akutmedizin steht daher weiter unter einer physischen, psychischen und sozialen Dauerbelastung. Da Schwangere durch direkte oder indirekte Folgen einer COVID-19-Erkrankung gefährdet sind, sollten diese - insbesondere bei Vorhandensein von Komorbiditäten (z.B. Diabetes mellitus) - als Hochrisikopopulation eingestuft und besonders geschützt werden.

Die meisten schwangeren Frauen (85-86\%), die an SARS-CoV-2 erkranken, weisen milde Krankheitsmerkmale auf. Dennoch werden schwere $(9,3-11,1 \%)$ bis sehr schwere Verläufe (2-6,9\%) beschrieben [1]. Die Wahrscheinlichkeit, dass SARS-CoV-2-positive Schwangere auf eine Intensivstation eingewiesen werden (10,5 vs. 3,9 pro 1000 Fälle; „adjusted risk ratio“ [aRR] 3,0; $95 \%$-Konfidenzintervall [95\%-KI] 2,6-3,4), eine invasive Beatmung $(2,9$ vs. 1,1 pro 1000 Fälle; aRR 2,9; $95 \%$-KI 2,2-3,8), eine extrakorporale Membranoxygenierung (ECMO) erhielten (0,7 vs. 0,3 pro 1000 Fälle; aRR 2,4; $95 \%$-KI $1,5-4,0)$ und verstarben ( 1,5 vs. 1,2 pro 1000 Fälle; aRR 1,7; $95 \%$-KI 1,2-2,4), war im Vergleich zu Nichtschwangeren signifikant erhöht [2].

In einer kürzlich publizierten SARSCoV-2-Studie konnte ein erhöhtes Erkrankungsrisiko bei Schwangeren und Neugeborenen nachgewiesen werden [3]. Die Analyse des Surveillance for Emerging Threats to Mothers and Babies Network (SET-NET) von 3912 Lebendgeburten zeigte, dass der Anteil der Frühgeburten $(<37$. Schwangerschaftswoche) mit $12,9 \%$ etwas über dem Durchschnitt der amerikanischen Bevölkerung von 10,2\% liegt. Bei der Mehrheit der SARS-CoV-2 positiven-Schwangeren trat eine Infektion im 3. Trimenon auf. Die Folge einer COVID-19-Infektion im 3. Trimester wiederum kann eine Frühgeburt sein.

In einer systematischen Übersichtsarbeit und Metaanalyse konnte ebenfalls ein erhöhtes Risiko für Frühgeburten von SARS-CoV-2-positiven Schwangeren nachgewiesen werden [4]. Die Wahrscheinlichkeit einer Frühgeburt bei COVID-19-Erkrankten war im Vergleich zu nichtinfizierten Schwangeren erhöht (OR 3,01; 95\%-KI 1,16-7,85). Zudem musste ein Viertel aller Neugeborenen, die von Müttern mit COVID19 geboren wurden, auf eine Neugeborenenstation aufgenommen werden (25\%) und hatte ein höheres Risiko bei Aufnahme (OR 3,13; 95 \%-Kl 2,05-4,78) als diejenigen, die von Müttern ohne COVID-19 geboren wurden.
Obwohl keine auffällige Häufung von Fehlbildungen in der Studie von Woodworth et al. [3] beobachtet wurde, sei darauf hingewiesen, dass dennoch bedingt durch hohes Fieber im Rahmen der COVID-19-Erkrankung während des ersten Trimenons der Schwangerschaft ein Risiko von Komplikationen und Fehlbildungen besteht. Fehlgeburten treten während der SARS-CoV-2Pandemie bisher nicht häufiger auf, die Datenlage ist aber für eine abschließende Beurteilung derzeit noch unzureichend.

Das Risiko einer perinatalen Infektion scheint eher gering. In der Studie von Woodworth et al. haben sich 16 von 610 Neugeborenen $(2,6 \%)$ mit positivem SARS-CoV-2-Testergebnis infiziert [3]. Zudem sei auf das erhöhte Risiko des Auftretens einer Thrombembolie unter COVID-19 hingewiesen [5]. Alleinig durch die Schwangerschaft besteht eine erhöhte Prothrombogenität, die im Rahmen einer zusätzlichen COVID-Erkrankung als additiver Risikofaktor potenziert wird.

Im Rahmen der COVID-19 Related Obstetric and Neonatal Outcome Study (CRONOS) wurden erstmalig Daten aus Deutschland vorgestellt [6]. Eingeschlossen werden Schwangere mit nachweislicher SARS-CoV-2-Infektion. Insgesamt 98 der 122 Kliniken beteiligen sich aktiv an der Registerstudie, die im vorigen Jahr 185.787 und somit 
$>20 \%$ der Geburten in Deutschland betreuten. Die Daten in dem Zeitraum vom 03.04.2020 bis zum 01.10.2020 ( $n=247$ Fälle von 65 Kliniken) wurden nun ausgewertet. Die Schwangeren waren vorwiegend jünger als 35 Jahre (7\%) und hatten einen Body-Mass-Index $(\mathrm{BMI})<30 \mathrm{~kg} / \mathrm{m}^{2}(79 \%) ; 38 \%$ der Frauen befanden sich in ihrer ersten Schwangerschaft. 14 der 247 Schwangeren, d.h. $5,7 \%$ der Fälle, wurden wegen COVID19 intensivmedizinisch behandelt. Bei der Mehrzahl der Frauen lagen keine speziellen Risikofaktoren vor. Fünf Schwangere waren entweder adipös, hatten hypertone Blutdruckwerte bei Aufnahme, einen bekannten Diabetes mellitus/ Gestationsdiabetes oder die Kombination aus diesen Risikofaktoren. $10 \mathrm{der}$ 14 Frauen infizierten sich im 3. Trimester. In 9 der 14 Fälle hat die SARS-CoV2-Infektion die Entscheidung zur Beendigung der Schwangerschaft oder den Geburtsmodus beeinflusst. 205 Frauen sind zwischenzeitlich von SARS-CoV-2 genesen und 185 haben entbunden. SARS-CoV-2 konnte bei 4 Neugeborenen $(2,2 \%)$ nachgewiesen werden, die alle in die häusliche Umgebung entlassen wurden. Eine Follow-up-Abfrage von 56 Familien nach der Neonatalzeit erbrachte keinen Fall einer neonatalen Neuinfektion. Obwohl die Daten der CRONOS-Studie für Deutschland einen überwiegend günstigen Verlauf einer Infektion mit SARS-CoV-2 nahelegen, scheint dennoch die Rate einer Frühgeburt im Vergleich zum bundesdeutschen Durchschnitt vergangener Jahre erhöht.

Die Aufsichtsbehörden der Bundesländer haben arbeitsmedizinische Empfehlungen zur Beschäftigung von schwangeren und stillenden Frauen im Zusammenhang mit dem Coronavirus SARS-CoV-2 bzw. der COVID-19-Erkrankung veröffentlicht. Am Beispiel der Veröffentlichung vom Ministerium für Arbeit, Gesundheit und Soziales des Lands Nordrhein-Westfalen [7] wird deutlich, dass Schwangere als Risikopersonen bzw. als besonders schutzwürdige Personen im Sinne des Mutterschutzgesetzes (MuSchG) eingestuft werden. Nach Mutterschutzgesetz ist der Arbeitgeber unter Hinzuziehung der/des Betriebsärztin/Betriebsarztes verpflich- tet, eine Gefährdungsbeurteilung des Arbeitsplatzes der Schwangeren vorzunehmen. Die Entscheidung über zu ergreifende Maßnahmen ist immer eine Einzelfallentscheidung.

Der Ausschuss für Biologische Arbeitsstoffe (ABAS), der das Bundesministerium für Arbeit und Soziales zu Fragen des Arbeitsschutzes bei Tätigkeiten mit biologischen Arbeitsstoffen berät, hat das Coronavirus SARS-CoV-2 aus präventiver Sicht in die Risikogruppe 3 nach der Biostoffverordnung eingestuft. Nach $\$ 11$ Absatz 2 MuSchG darf der Arbeitgeber eine schwangere Frau u.a. keine Tätigkeiten ausüben lassen und sie keinen Arbeitsbedingungen aussetzen, bei denen sie mit Biostoffen der Risikogruppe 3 in einem Maß in Kontakt kommt bzw. kommen kann, die für sie oder ihr Kind eine unverantwortbare Gefährdung darstellt. Dies ist im Rahmen der Gefährdungsbeurteilung im Einzelfall abzuklären [7].

Vor dem Hintergrund, dass die Auswirkungen einer SARS-CoV-2-Infektion derzeit noch nicht zuverlässig bewertet werden können, ist ein gegenüber der Allgemeinbevölkerung erhöhtes Infektionsrisiko mit SARS-CoV-2 am Arbeitsoder Ausbildungsplatz aus präventiven Gründen als unverantwortbare Gefährdung einzustufen. Dies gilt nach Auffassung des Ausschusses für Mutterschutz [8] beim Bundesfamilienministerium und basierend auf den Empfehlungen der Aufsichtsbehörden der Bundesländer nicht nur beim Umgang mit Erkrankungsfällen (laborbestätigten COVID19-Fällen) oder ärztlich begründeten Verdachtsfällen, sondern bereits bei Kontakt zu ständig wechselnden Personen oder bei regelmäßigem Kontakt zu einer größeren Anzahl von Personen [7]. Eine Weiterbeschäftigung einer schwangeren Frau darf nur insoweit erfolgen, als durch effektive Schutzmaßnahmen sichergestellt ist, dass die schwangere Frau am Arbeitsplatz keinem höheren Infektionsrisiko ausgesetzt ist als die Allgemeinbevölkerung [7].

Die Gefährdungsbeurteilung sollte allgemeine und spezielle Kriterien berücksichtigen.

Allgemeine Kriterien im Rahmen der Gefährdungsbeurteilung sind:
- Welcher Art und Häufigkeit sind die Kontakte sowie die Zusammensetzung der Personengruppe? Besteht ein Kontakt zu ständig wechselnden Personen/Patienten/Publikum?

- Kann ein Mindestabstand von 1,5 m zu anderen Personen sicher eingehalten werden?

- Ist ein enger Kontakt im Rahmen eines persönlichen Gesprächs („Faceto-face“-Patientengespräch) unvermeidbar und dauert dieser länger als $15 \mathrm{~min}$ ?

- Besteht Umgang mit an den Atemwegen erkrankten oder krankheitsverdächtigen Personen?

- Werden Tätigkeiten durchgeführt, die mit einer erhöhten Aerosolbildung einhergehen?

- Wie sieht es mit der Umsetzung der Hygienestandards und der Versorgung mit persönlicher Schutzausrüstung beispielsweise mit Atemschutzmasken in der vorliegenden Belastungssituation zum jetzigen Zeitpunkt aus?

- Wie sind die Raum- und Lüftungsverhältnisse am Arbeitsplatz?

Besondere Kriterien im Gesundheitswesen, in der Pflege und in ähnlichen Bereichen sind:

- In diesen Bereichen stellt der vom Mutterschutzgesetz geforderte Ausschluss einer unverantwortbaren Gefährdung eine arbeitsorganisatorische Herausforderung dar. Denn hier sind regelmäßige Kontakte zu ständig wechselnden Personen bzw. einer größeren Anzahl von Personen häufig.

- Schwangere Frauen dürfen grundsätzlich keine Tätigkeiten an sog. Verdachtsfällen oder infektiösen Patienten oder auch infektiösen Materialien wie beispielsweise Laborproben verrichten.

- Angesichts der sich dynamisch entwickelnden Infektionslage sollten Schwangere bevorzugt mit patientenfernen Tätigkeiten in gut belüftbaren Räumen beschäftigt werden.

- Tätigkeiten mit Patientenkontakten sind nur nach individueller Gefährdungsbeurteilung mit Charakterisierung der Patienten möglich, 
insbesondere müssen sie frei sein von Symptomen einer aerogen übertragbaren Infektionserkrankung und in der Lage sein, einen Mund-Nasen-Schutz zu tragen. Die Patienten müssen bezüglich SARS-CoV-2 voruntersucht sein, es muss eine strenge Besucherregelung umgesetzt werden. Eine hohe Fluktuation ist $\mathrm{zu}$ vermeiden. Dies kann in stationären Bereichen umsetzbar sein, jedoch meist nicht im ambulanten Setting.

- Tätigkeiten mit engem Körperkontakt zu Patienten, insbesondere in Nähe zum Kopf, und Tätigkeiten, die zu Aerosolbildung führen können, sind nicht möglich.

- In Abhängigkeit von der Gefährdungsbeurteilung ist das temporäre Tragen einer FFP2-Maske für Schwangere möglich, wenn regelmäBig Tragepausen ermöglicht werden. Nach Beschluss 609 des ABAS [9] ist in Einrichtungen des Gesundheitsdiensts davon auszugehen, dass sich die Maskenfilter beim Tragen nicht durch Stäube zusetzen, der Einatemwiderstand also im Normbereich bleibt.

\section{Korrespondenzadresse}

\section{Prof. Dr. med. Guido Michels}

Klinik für Akut- und Notfallmedizin, St.Antonius-Hospital Eschweiler, Akademisches Lehrkrankenhaus der RWTH Aachen Dechant-Deckers-Str. 8, 52249 Eschweiler, Deutschland

guido.michels@sah-eschweiler.de

\section{Einhaltung ethischer Richtlinien}

Interessenkonflikt. G. Michels und U. Ochmann geben an, dass kein Interessenkonflikt besteht.

Für diesen Beitrag wurden von den Autoren keine Studien an Menschen oder Tieren durchgeführt. Für die aufgeführten Studien gelten die jeweils dort angegebenen ethischen Richtlinien.

\section{Literatur}

1. Api O, Sen C, Debska M et al (2020) Clinical management of coronavirus disease 2019 (COVID19) in pregnancy: recommendations of WAPMWorld Association of Perinatal Medicine. J Perinat Med 48(9):857-866
2. Zambrano LD, Ellington S, Strid P et al (2020) Update: characteristics of symptomatic women of reproductive age with laboratory-confirmed SARS-coV-2 infection by pregnancy status-United States, january 22-october 3, 2020. MMWR Morb Mortal Wkly Rep 69(44):1641-1647

3. Woodworth KR, Olsen EO, Neelam V et al (2020) Birth and infant outcomes following laboratory-confirmed SARS-coV-2 infection in pregnancy-SET-NET, 16 jurisdictions, march 29-October 14, 2020. MMWR Morb Mortal Wkly Rep 69(44):1635-1640

4. Allotey J, Stallings E, Bonet M, Yap M, Chatterjee S, Kew T et al (2020) Clinical manifestations, risk factors, and maternal and perinatal outcomes of coronavirus disease 2019 in pregnancy: living systematic review and meta-analysis. BMJ 370:m3320

5. Ahmed I, Azhar A, Eltaweel N, Tan BK (2020) First COVID-19 maternal mortality in the UK associated with thrombotic complications. Br J Haematol 190:e37-e38

6. Pecks U, Kuschel B, Mense $L$, Oppelt $P$, Rüdiger $M$ (2020) CRONOS-Netzwerk: Pregnancy and SARS CoV-2 infection in Germany-the CRONOS registry. Dtsch Arztebl Int 117:841-842

7. https://www.mags.nrw/sites/default/files/asset/ document/arbeitsmedizinische_empfehlungen_ musch_und_corona.pdf;. Zugegriffen: 17. Dez. 2020

8. https://www.bafza.de/fileadmin/Programme und_Foerderungen/Unterstuetzung_von_ Gremien/Ausschuss-fuer-Mutterschutz/ Informationspapier_Mutterschutz_und_SARSCoV-2_200414.pdf;.Zugegriffen: 17.Dez. 2020

9. https://www.baua.de/DE/Angebote/Rechtstexteund-Technische-Regeln/Regelwerk/TRBA/pdf/ Beschluss-609.pdf?_blob=publicationFile\& v=49;.Zugegriffen: 17. Dez. 2020
Hier steht eine Anzeige. Springer 